L'HOMME L'Homme

185-186 | 2008

L'anthropologue et le contemporain : autour de Marc Augé

\title{
Actualité du paganisme
}

Et contemporanéité des prophétismes

\section{André Mary}

\section{(2) OpenEdition \\ Journals}

Édition électronique

URL : http://journals.openedition.org/lhomme/24195

DOI : 10.4000//homme.24195

ISSN : 1953-8103

Éditeur

Éditions de l'EHESS

Édition imprimée

Date de publication : 1 janvier 2008

Pagination : 365-386

ISSN : 0439-4216

Référence électronique

André Mary, «Actualité du paganisme », L'Homme [En ligne], 185-186 | 2008, mis en ligne le 01 janvier 2010, consulté le 19 avril 2019. URL : http://journals.openedition.org//homme/24195 ; DOI : 10.4000/ Ihomme.24195

(c) École des hautes études en sciences sociales 


\title{
Actualité du paganisme et contemporanéité des prophétismes
}

\author{
André Mary
}

$\mathrm{R}_{\mathrm{r}}$ actuel des néopaganismes en tout genre (néo-indien, néo-chamanique ou néo-celtique) réveillés entre autres par la mouvance New Age. Il est possible en effet de repérer, au-delà de l'imaginaire enchanté du rapport au monde des esprits, quelque "dimension païenne" dans tel ou tel trait de ces formes de la religiosité dite "surmoderne ": l'interprétation des événements-signes de la vie personnelle, le syncrétisme des références fragmentaires ou la consommation clientéliste et pragmatique des offres cultuelles (in Bessis 2004 : 48). Mais ce comparatisme analogique gomme un peu vite le caractère systématique du socle anthropologique (à trois pieds) que représente la "logique païenne" pour l'auteur du Génie du paganisme (Augé 1982). Le paganisme, conçu au départ comme l'idéologique des sociétés lignagères, mais pensé aujourd'hui comme la matrice rituelle de toute vie sociale, a été particulièrement mis à l'épreuve dans le cadre des prophétismes africains qui s'offrent comme situations-limites de confrontation avec son contraire, le christianisme missionnaire. La mise en péril des catégories de cette logique païenne, sur fond de relation duelle, change d'échelle avec le phénomène actuel des pentecôtismes, notamment indigènes, et leur propre veine prophétique. L'irruption des prophètes pasteurs, qui prennent le relais des prophètes guérisseurs, relance la question de la pertinence idéologique et anthropologique du paganisme.

\section{De l'actualité à la contemporanéité}

L'actualité, au sens fort du terme dans l'esprit du geste épistémologique de Sociologie actuelle de l'Afrique Noire (Balandier 1955) n'est pas, comme le souligne Marc Augé, une concession à la sociologie de l'air du temps, 
"c'est précisément la totalité des déterminations et des expressions d'un champ social à un moment donné» (Augé 1979 : 175). Cette exigence de totalité et de systématicité, Georges Balandier l'a trouvée, à un moment donné, dans la «situation coloniale». Les révélateurs, les leviers, de ce changement d'échelle par rapport à l'ethnie des ethnologues étaient ces objets "nouveaux", polémiques et hors-jeu, que représentaient dans les années 1940-1950 les syncrétismes et les prophétismes. Pour comprendre des phénomènes comme le bwiti syncrétique du Gabon ou le prophétisme kimbanguiste du Congo, il était impossible de s'en tenir aux traits sociaux et culturels d'une ethnie fang ou kongo, à leur diffusion dans une aire culturelle régionale; il fallait changer le cadre et le niveau d'analyse, penser en termes de "situation ». Placer la "logique païenne» ou l'« idéologique lignagère ", au cœur de l'exigence de compréhension structurale des situations ivoiriennes des années 1960 et 1970, relevait sans doute d'un même souci d'actualité, mais les prophétismes, et encore moins les syncrétismes, n'étaient plus de façon aussi évidente, vus du rivage alladian, des analyseurs d'un monde en train de se faire: ces productions de sens se comprennent dans la continuité des ressources de la matrice du paganisme, de ses dérives ou de ses impasses, au mieux comme des révélateurs en négatif du malin génie du paganisme.

Le paradigme de la "contemporanéité" conduit à une réévaluation du phénomène prophétique. Il s’inscrit à la fois dans le prolongement du geste épistémologique de Georges Balandier - la colonisation offrant une anticipation de l'actualité de la mondialisation - et resitue dans le même temps la place accordée à la figure prophétique. Qu'il n'y ait pas de malentendus! Le paganisme est toujours là, c'est le socle anthropologique, et les ambiguïtés et ambivalences des prophétismes sont plus que jamais soulignées, y compris dans leurs versions les plus récentes, mais le changement de perspective est significatif: le prophète n'est plus condamné à être un simple avatar du paganisme, il devient un "anticipateur» de la mondialisation, un « expérimentateur» idéologique, un «bricoleur de sens $»^{1}$ (in Bessis 2004 : 113). Une des clés de ce changement de regard sur l'histoire prophétique ivoirienne et mondiale est entre autres (car il y a aussi le détour par les messianismes des Amériques noires et indiennes) le «retour de Harris ", notre contemporain: "Le mythe de Harris fait aujourd'hui partie de l'histoire ivoirienne. Mais les cultes prophétiques n’en constituent qu'une série d'échos affaiblis» (Augé 1994: 153). Nul doute en effet que Harris est devenu, avec le recul, le paradigme du prophétisme contemporain.

1. Le paradigme du bricolage " au sens noble du terme " est largement repris dans le "dialogue " cité, mais si le "sens noble " est bien celui de la tradition anthropologique héritée de Claude LéviStrauss (1962), il est plutôt mal venu d’assimiler «ingénieur du sens » et "bricolage de génie ». 
L'enjeu n'est pas seulement mythique. Il est d'abord historiographique. Nous savons mieux aujourd'hui à quel point l'histoire coloniale participe de l'histoire mondiale. L'osmose précoce entre colonisateurs et colonisés, l'inversion des rôles, des colons noirs américains aux missionnaires indigènes, tout au long du XIXe siècle, au sein des mondes anglophones (Liberia, Nigeria), obligent à systématiser le principe de contemporanéité en y introduisant plus de symétrie. La rencontre entre des missionnaires protestants projetant sur l'Afrique une utopie de retour aux vertus d'un monde rural éloigné des hérésies des Lumières et des dérives du modernisme industriel, et des Nègres lettrés cherchant dans le monde tribal du judaïsme une source d'identification et dans la trame biblique de l'exil une voie de salut pour leur peuple, ne correspond ni au scénario de l'irruption soudaine d'un monde extérieur, ni à la cohabitation de deux mondes séparés. Elle associe la reconnaissance du même dans l'autre et les malentendus d'un monde commun ${ }^{2}$.

Harris, cet instituteur et catéchiste glebo du Liberia qui vit à partir de sa conversion en 1880 chaque événement de sa vie quotidienne sur fond de verset biblique, est un authentique contemporain de Blyden (Shank 1994 : 70), l'exemple type du "métis planétaire " qui cherchait déjà dans les années 1880 une synthèse entre l'islam et le christianisme pour fonder une identité religieuse de la race noire. Harris, qui porte dans son costume de prophète les emblèmes de ce synthétisme religieux (turban musulman, tunique blanche et crosse romaine), est plus particulièrement le prototype de ces lettrés «indigènes » travaillés par les ambivalences de la posture de "colonisé civilisé", se précipitant, dans sa révolte et son emprisonnement, en situation limite, dans la transe visionnaire et l'aventure prophétique. Il est aussi le "frère en Christ" de cette génération de returnees, de ces fils d'esclaves libérés de Sierra Leone et du Liberia qui découvrent leur identité africaine (yoruba ou autres) dans le mouvement même de leur conversion au christianisme méthodiste ou anglican. L'allochronisme apparent de la geste de ce passeur de frontières, the Black Elijah, au regard de la tradition ivoirienne des petits prophètes, à plusieurs décades d'intervalle, mérite réflexion, si l'on veut donner tout son sens à la contemporanéité des prophétismes "ivoiriens" aussi bien qu'au processus de mythologisation de la figure d'Harris dans l'ensemble des christianismes de l'Afrique de l'Ouest.

2. Voir à ce sujet, entre autres, Peel 2000. Il est significatif que le précurseur de la notion de coevalness soit l'auteur d'une monographie célèbre consacrée à un mouvement charismatique contemporain, Jamaa, initié de son vivant par le père Tempels, celui qui inventait dans le même temps une ethnophilosophie bantoue particulièrement «allochronique» (Fabian 1971). 
Une des figures de la première génération des prophètes harristes ivoiriens (Dozon : 1995), Papa Nouveau - le paradigme du prophète en son pays, un "prophète pur» selon Houphouët-Boigny -, est mort dans son village en 2001. La même année, Kacou Séverin - « Prophète de toutes les nations », le profil type des "prophètes-pasteurs " évangéliques et ivoiriens meurt également, dans un accident tragique, une mort "christique» et sacrificielle, un Vendredi Saint (Mary 2002). En prophétisant, comme bien d'autres, l'élection d'un président "chrétien " (born again), agent du salut du peuple ivoirien martyrisé, ces prophètes reprennent à leur façon l'effet d'annonce des prophètes paysans sur les promesses du "miracle ivoirien » et du régime du " président planteur ». Dans la Côte-d'Ivoire des années 2000, face aux déclins des "petits prophètes " et aux divisions de l'Église harriste, le réveil prophétique passe de fait par les nouveaux "prophètes pasteurs ", d'inspiration évangélique, à la fois fortement ivoiriens et internationaux, qui font la une de la presse et des médias, et participent activement à l'actualité politique jusqu'à jouer les conseillers de la nouvelle " gouvernance ». Ces «véritables prophètes » font explicitement retour à la "veine prophétique» d'Harris, particulièrement au message biblique et messianique de la culture méthodiste et pentecôtiste dont il était fortement nourri et inspiré (Shank 1994). Les prophètes "pressés ", de la ville, des aéroports et de l'international, anciens étudiants de l'université de Cocody ayant séjourné en Europe ou aux États-Unis, sont des «envoyés de Dieu » porteurs d'une vision de salut pour toutes les nations.

Dans l'esprit du retour à la primauté de la vocation prophétique, ce que les auteurs américains des années 1980 (Bill Hamon, Peter Wagner) appellent le «moment prophétique ", le cinquième âge de la Révélation après la Réforme, celui qui réveillera de nouveaux ministères enfouis dans les heures sombres de l'Église, ces prophètes visent moins la fondation d'une Église que l'animation d'un ministère itinérant situé au delà de toutes les dénominations ou confessions particulières, laissant à la charge de quelques apôtres les relais locaux de leur entreprise. Ils contribuent ainsi objectivement, en reprenant chacun pour leur compte le geste prophétique, à la démultiplication de ce que les observateurs et les journalistes appellent les « sectes protestantes ».

\section{Anthropologie païenne et altérité chrétienne}

Si les prophètes de l'époque coloniale anticipent d'une certaine façon la nécessité d'avoir à penser l'inscription des autres, des Blancs et des Noirs, dans une même temporalité et dans un même monde, les prophètes de la «mondialisation» n'échappent pas aux contradictions de l'entreprise. 
Ce n'est pas par hasard que Gérard Althabe ${ }^{3}$ illustrait les paradoxes de la "contemporanéité " en se référant au processus de planétarisation du christianisme et de multiplication des "christianités", un mouvement particulièrement observé dans le cadre de la protestantisation des mondes sud-américains. La mouvance évangélique et pentecôtiste illustre en effet l'image d'une religiosité charismatique globale, émergeant au début du $\mathrm{XX}^{\mathrm{e}}$ siècle, avec une étonnante simultanéité d'un bout à l'autre de la planète, dans une temporalité à peine différée (aux États-Unis, aussi bien qu'en Afrique du Sud ou au Brésil), se développant avec une accélération sans précédent dans l'histoire des religions, notamment par le recours aux médias, et faisant appel à la puissance de l'esprit comme solution immédiate et miraculeuse de tous les problèmes. Dans le même temps, ce monde commun de "convertis» (des crente du Brésil aux pente d'Éthiopie), témoigne d'une plasticité étonnante qui fait place à une multiplicité d'identités séparées et reterritorialisées, conjuguant l'ethnicité, le racialisme et le nationalisme biblique, le "parler en langues » et le langage du corps. Cette logique de déterritorialisation et de reterritorialisation qui se déploie à l'échelle planétaire, principalement dans les périphéries urbaines, alterne les grands rassemblements dans les stades et les groupes de prière des "églises de maison».

Mais le paradoxe le plus évident, au regard de notre propos, c'est la manière dont le prophétisme pentecôtiste "réveille", si l'on peut dire, le "paganisme", comme catégorie du discours et de la pratique religieuse, et lui redonne une étonnante actualité. La montée en puissance de la sorcellerie et du fétichisme qui étaient au fondement de l'action des prophètes guérisseurs, loin d'être renvoyés à l'obscurantisme des mondes paysans, refait plus que jamais surface au cœur de la modernité religieuse «avancée ", avec la caution des pasteurs américains. La délivrance des démons n'était déjà plus, au cœur de l'enclos prophétique, une simple affaire villageoise d'accusation et de conjuration familiale, mais la diabolisation de la sorcellerie entraîne un déplacement significatif à l'échelle mondiale des enjeux individuels dans une guerre spirituelle contre le malin et ses ruses. Le paradoxe est à son comble quand des cultures qui ont rompu avec leurs coutumes païennes où des malades qui sont totalement ignorants ou incultes en matière de sorcellerie se voient "enseigner" les secrets de ces choses par des pasteurs qui produisent et diffusent à leur compte des traités de sorcellerie et de démonologie. Le cercle bien connu du prophète et

3. Manuscrit inédit sur le projet de fondation du Centre d'anthropologie des mondes contemporains, de l'École des hautes études en sciences sociales (1993). 
du sorcier se retrouve dans cette complicité objective et cultivée entres les pasteurs et les esprits diaboliques.

La relation duelle avec les démons du paganisme dont le scénario se répète, depuis l'époque missionnaire, dans les prophétismes aussi bien que dans les pentecôtismes, oblige à revenir sur les termes en présence et surtout à interroger ces enjeux anthropologiques. Le paganisme n'est pas en effet, ni pour Marc Augé, ni pour les autres, chrétiens ou païens, une "religion », et le "génie du paganisme » se défend d'être la réhabilitation savante d'une antireligion stigmatisée par le christianisme. Dans les versions théologiques qui en sont données, c'est sans doute le jeu des écarts et des inversions qui prime: "le paganisme est le contraire du christianisme " (Augé 1982: 14). On connaît les deux « catéchismes » en présence et surtout la difficulté qu'il y a à surmonter les pièges des oppositions signifiantes qui président à la stigmatisation de l'autre, et au jeu de l'inversion des valeurs. L'auteur du Génie du paganisme n'est pas le dernier à dénoncer l'ethnocentrisme qui caractérise ces opérations de réhabilitation d'une religion du Livre à l'envers (Augé 1979: 25). Défiant toute analyse structurale, le paganisme a pour caractéristique fondamentale de n'être pas dualiste. Sa plasticité légendaire va de pair avec un syncrétisme consubstantiel qui pratique le cumul, l'addition ou l'alternance, mais ignore la synthèse, et encore plus le souci du dépassement (Augé 1982 : 14). C’est cette pratique du mélange incongru qui a toujours fait du paganisme, aux yeux du christianisme, l'autre de la vraie religion, sa perversion, sachant que le sens de la frontière passe par bien des compromis qui reconnaissent que l'autre païen est dans la place et qu'on en n'a jamais fini avec lui. Dans l'histoire du christianisme missionnaire, le paganisme a été identifié à la religion archaïque des mondes primitifs, paysans ou populaires, mais il en est venu à l'inverse à épouser toutes les dérives de la modernité. Dès la fin du XIX ${ }^{e}$ siècle, la dénonciation du paganisme se conjugue avec la critique du modernisme et des errements du rationalisme et du matérialisme.

Le "génie du paganisme " n'est donc ni la religion des autres, telle qu'ils la pensent eux-mêmes, ni une antireligion stigmatisée ou réhabilitée, c'est une anthropologie qui trouve à s'exprimer, entre autres par le biais des choses religieuses (mais pas seulement), dans une certaine forme de rapport au monde, aux autres et à soi. La référence déterminante ici est la place centrale (et croissante) accordée au rituel. Le religieux ritualisé, avec son ancrage dans des communautés "naturelles", son attachement à des lieux et des dieux-objets, son "épistémé de l'immanence " (Augé 1997 : 66), et surtout ses dispositifs de médiation symbolique, confine à la reproduction machinale et ordinaire du lien social. Cette sacralité rituelle s'inscrit 
en tension avec une religiosité personnelle, spirituelle et éthique, que les religions monothéistes et universelles n'ont cessé de privilégier sans pour autant réussir à résorber ou éradiquer l'autre, le contraire, l'ennemi par excellence : le rituel (Augé 1994 : 121).

Deux questions de fond se posent ici à l'anthropologue des religions. La dissymétrie de ces deux rapports aux choses religieuses est frappante. L'épaisseur anthropologique et sociale du paganisme est telle que l'idée d'une matrice anthropologique "chrétienne" (sans parler de l'islam) se présente sur le terrain des sociétés occidentales ou africaines, comme un concept limite, une anthropologie "négative", à moins qu'elle ne laisse place à la théologie: "ce qui n’est pas païen, c'est la spéculation sur le devenir, sur la personne au-delà de la mort, ou sur la notion de péché... » (in Bessis 2004 : 48). L'anthropologie des sociétés chrétiennes ne prendelle sens qu'au regard d'une anthropologie du rituel et de sa matrice païenne? Marc Augé stigmatise la façon dont les analyses contemporaines font de l'archaïque, l'impensé par excellence (Augé 1994: 120), mais l'argument peut facilement se retourner : le chrétien n'est-il pas l'impensé qui rend dicible et pensable le socle anthropologique du paganisme? L'autre en définitive serait le chrétien ${ }^{4}$.

À vrai dire, la dissymétrie s'étend à la relation elle-même, et c'est bien dans un dialogue avec la sociologie contemporaine du croire que l'anthropologue du paganisme nous dit le fond de sa pensée : "la religion qui s'autonomise dans la modernité chrétienne n'a rien à voir avec ce qu'on désigne comme "religion" lorsqu'on fait référence globalement à l'ensemble des sociétés païennes" (Ibid.: 120). L'affirmation prend acte du " tournant» historique que constitue l'émergence des religions universalistes, mais la conclusion va plus loin : "l'apparition et le développement d'une telle religion [universaliste] se surajoutent à l'existant et ne l'annulent pas. Le rapport ritualisé au monde continue notamment dans la sphère politique...» (Ibid., je souligne). Le syncrétisme, compris comme logique du cumul et de l'addition, est décidément consubstantiel à la logique païenne et constitue le ressort de toute sa force englobante (pour ne pas dire dévorante), celle qui permet à cette matrice symbolique de persister, même dans un monde désenchanté, en épousant le social.

4. Dans une confession d'incroyance qui témoigne d'une indifférence «totale, animale et définitive ", parfaitement respectable, vis-à-vis de l'enjeu du croire au sens chrétien, il est clair que l'altérité la plus incompréhensible, chez l'autre proche, ce n'est pas d'aller à la messe, ou d'adhérer au dogme, mais de soutenir que l'essentiel c'est la "foi personnelle, raisonnée, intime, que sais-je?» (Augé 1986: 23). 


\section{Imaginaire pentecôtiste et réveil du paganisme}

372 Le fait anthropologique majeur c'est le mélange des formes de religiosité, la cohabitation ou le recouvrement troublant du même et de l'autre. Mais au regard du refus du paganisme, les religions monothéistes ne sont pas toutes à la même enseigne. Le ritualisme païen du catholicisme, cette religion de l'appartenance héritée, son culte des lieux et des objets consacrés, son recours aux saints intermédiaires, en un mot cette religion du sacré, est en tension avec la religiosité de la foi personnelle et de l'intimité de la relation éthique à un Dieu transcendant que met en avant la tradition protestante. Les pentecôtismes, notamment brésilien ou africain, ont fait de la chasse au paganisme et au fétichisme du catholicisme leur cheval de bataille. Mais la référence à un christianisme essentiellement éthique et personnel ne peut être que troublée par l'imaginaire du mal que véhicule dans le même temps toute la culture pentecôtiste à propos des œuvres de Satan ou du Diable et le recours aux pratiques de l'exorcisme. Comment cet univers qui se dit avec force chrétien, qui ne jure que par la Bible et par Jésus, peut-il adhérer pleinement à la lettre du catéchisme païen : «La conscience persécutrice du mal, le sens de la force, l'immanence du monde divin au monde humain" (Augé 1982: 72) ? La figure de l'autre persécuteur est omniprésente dans la prédication sous la forme de Satan ou du diable, qui se déploie en toute logique païenne en une multiplicité illimitée d'esprits diaboliques qui vous possèdent de l'intérieur autant qu'ils se "manifestent ». La "puissance du sang de Jésus » est ce qui fait l'efficacité de la protection et de la solution qu'il apporte à vos problèmes. La vision du prophète pasteur nous plonge dans un univers totalement dominé par le combat des forces spirituelles et la victoire attendue de la puissance de l'esprit. Enfin l'expérience de la conversion comme "effusion de l'esprit », les charismes du rêve, de la vision, de la prophétie, autant que les miracles de la guérison spirituelle, témoignent d'une présence permanente du monde divin au monde humain et d'un enchantement quotidien.

Le pentecôtisme à sa façon se veut plus qu'une nouvelle religion, c'est aussi une autre culture et un autre rapport aux choses religieuses, qui ne manquent pas de scandaliser en premier les représentants des religions établies confrontés à cette religion de "parvenus». Trois exemples parmi d'autres. La conversion ici n'est pas présentée comme une démarche "spirituelle» de quête de sens, l'adhésion à une doctrine théologique : le pasteur vous invite avant tout à dire et à résoudre vos problèmes les plus immédiats et les plus quotidiens, tous vos problèmes (maladie, stérilité, chômage, carte de séjour). La citation des versets de la Bible est mise au 
service de l'élucidation des situations de vie. L'expérience qui vous est proposée (la rencontre et l'acceptation de Jésus) n'est rien d'autre que la manifestation visible, tangible, matérielle de la puissance de l'esprit, et suppose, dans les cas difficiles, un véritablement engagement dans une épreuve de force. Bien sûr, il faut payer, et encore payer, parce que tout se paie, on ne reçoit rien si on ne donne pas, répètent les pasteurs, et plus on donne, plus on a des chances de recevoir. La surenchère dans le don prend des allures de potlatch, il s'agit bien de mettre Dieu au défi, de faire un pari. La liturgie de l'argent et du business est omniprésente, indécente, mais sans complexe. Pour la "théologie de la prospérité ", un bon chrétien n’est pas pauvre, c'est un gagnant et cela se montre par des dépenses ostentatoires. Cette religiosité pragmatique nous éloigne des valeurs de l'ascétisme protestant, de l'évangile du travail et de l'angoisse de l'élection.

La posture de la rupture affichée par rapport aux religions établies, sur fond de relation en miroir, exigerait des analyses plus nuancées qui tiennent compte de la multiplicité et de l'hybridité des pentecôtismes nationaux ou indigènes. Dans sa dénonciation du paganisme catholique, le pentecôtisme brésilien ne s'affirme-t-il pas comme antiritualiste et antisyncrétique? Les pasteurs de l'Église universelle font scandale en brisant les idoles du culte des saints et en s'attaquant au noyau de la sacralité catholique (le fameux "coup de pied à la Vierge»). Cette "religion de l'émotion" et de l'expression spontanée de la ferveur religieuse collective refuse la pompe catholique et toute liturgie sacrale ordonnée et codifiée. Il serait néanmoins difficile d'ignorer les dispositifs rituels qui prennent le relais aussi bien dans les chants de louange que dans les corps à corps des séance de délivrance. Les manifestations spectaculaires et orchestrées de la puissance de l'esprit n'encouragent pas une religion de l'intériorité. À l'inverse, on évoque souvent, à propos des guérisons divines, des miracles quotidiens et des démonstrations de la puissance de l'esprit, un retour en force de la dimension "magico-religieuse », mais le caractère manifeste de cette puissance spirituelle fait l'économie des médiations ordinaires de l'efficacité symbolique. L'imposition des mains ou l'huile de l'onction (comme la canne-croix de Harris) ne sont là que pour transmettre une force qui vient de Dieu seul.

\section{L'entre-deux prophétique}

Cette relation d'inversion / perversion par rapport aux cultes païens et à la religion catholique bouscule les évidences de la modernité religieuse et suggère quelque parallèle avec la logique de l'entre-deux développée par 
Marc Augé à propos des avatars du rituel prophétique (1997: 114) ${ }^{6}$. Les relectures successives du sens des prophétismes jouent en effet sur un continuum situé entre deux figures opposées qui ne cessent de se télescoper ou de s'entrecroiser. La première figure, qu'incarnerait plutôt Odjo, et à sa façon Atcho, recourt à une "grille prophétique" de lecture de l'événement qui mise sur la force toujours prégnante d'une logique lignagère quelque peu dévoyée: "des éléments épars de modernité sont absorbés par le seul univers de sens qui tienne un peu le coup face au caractère discontinu, baroque et souvent injuste du monde officiel " ${ }^{7}$ (Augé \& Colleyn 1990 : 19). Mis à part des échos fragmentaires issus de la prédication missionnaire, la grille biblique est globalement étrangère à ce monde, à l'image d'un Papa Nouveau qui interdisait à ses disciples d'ouvrir la Bible pour autant parfaitement exposée. Les problèmes de l'individu (maladie, stérilité, chômage, échec scolaire) trouvent leur solution dans les lignes de force de la structure familiale, lieu par excellence de l'élucidation des affaires de sorcellerie. Le prophète guérisseur n'hésite pas d'ailleurs à convoquer les familles pour faciliter les aveux et encourager le pardon. Les dispositifs de confession, nous y reviendrons, sont censés lever les contradictions du "moi divisé » entre les aspirations individuelles et la force des liens familiaux. La retraite prolongée dans l'enclos du prophète se déroule sous le signe d'une re-communautarisation qui opère un déplacement par rapport à la dépendance familiale: le malade devient infirmier avant le retour attendu à la vie active.

La figure du "véritable prophète», envoyé de Dieu, qu’incarne Harris s'inspire en revanche de l'idéologie de l'élection et de la révélation divine propre à la trame biblique. Élu personnellement par un Dieu unique qui lui révèle sa mission, il ne peut qu'être un agent d'individualisation invitant à la rupture avec la logique lignagère. Témoin dans sa vie des contradictions engendrées par l'accélération de l'histoire, il inscrit les problèmes de chacun dans une vision globale du devenir de la race noire promue au rang de peuple élu de Dieu par sa médiation. L'annonce de l'imminence des temps nouveaux où les Noirs seront enfin tous des Blancs va de pair avec la gestion de l'attente différée dans quelque hors-lieu de vie communautaire (une Jérusalem céleste) coupé des territorialités ethniques et villageoises.

Entre ces deux pôles du continuum prophétique se dessine une figure de l'entre-deux qui pourrait relever de ce que Marc Augé appelle l'entredeux mythique (1997 : 114). Dans sa version diachronique, cette figure se

6. Sur cette «logique de l'entre-deux », voir également Mary 1993.

7. Souligné par nous. 
construit dans le mouvement qui conduit le passeur de frontières, porteur d'une vision et chargé d'une mission divine pour toutes les nations, aux petits prophètes en leur pays, repliés sur leur enclos villageois et les ressources de leur environnement familial. Figure régressive, elle apparaît comme une retombée ou un épuisement de la veine prophétique, à l'image de la geste de Harris qui finit lui-même, dans l'impasse, par négocier quelque compromis avec les diverses Églises qui réclament sa caution. Mais le mouvement en retour d'appropriation de la figure d'Harris par les petits prophètes, les versions légendaires de ses engagements dans les rapports de force des mondes qu'ils traversent, les adaptations locales de son "message " aux nations peuvent aussi se lire comme un enrichissement (et même une invention) de la mythologie harriste. Cette figure fragmentaire et double traduit les ambivalences et les ambiguïtés qui ont conduit ses disciples à percevoir l'homme de Dieu comme un "homme fort ». Bien plus, le rituel prophétique auquel participent depuis un siècle les prophètes ivoiriens obéit comme tout rituel à une logique du recommencement, et chaque itinéraire prophétique participe à la fois de la répétition du même scénario et du bricolage de nouvelles cosmologies ou de nouveaux dispositifs rituels. Les figures intermédiaires entre les prophètes les plus païens et les plus «chrétiens » n'ont cessé de se démultiplier dans un même espace d'entre-deux mythique balisé par le jeu des aller-retour entre la force du Dieu unique et les ressources des ancêtres lignagers, le message aux nations et la réethnicisation du territoire, l'appel au tribunal de la conscience et le recours au schéma persécutif.

Le schéma diachronique qui mène, pour résumer, de Harris à Odjo, dans l'attente de quelque retour au paradigme perdu de Harris et à sa vocation anticipatrice et libératrice n'exclut donc pas une lecture plus synchronique (et plus symétrique, sinon syncrétique). La figure du prophète de l'entredeux tire toute sa force (et non sa faiblesse) de l'ambivalence du personnage (à la fois chrétien et païen) et de l'ambiguïté de son entreprise (ni chrétienne, ni païenne) à distance de tout souci de synthèse ou de dépassement. Les " véritables prophètes de notre contemporanéité ", dont le paradigme serait alors plutôt le prophète Atcho (Augé 1982 : 247), ne sont-ils pas ceux qui font écho aux contradictions qui travaillent les individus et qui inventent des dispositifs rituels de médiation qui fonctionnement " en double », sur la logique de la double entente, le bon usage de l'ambivalence et la richesse des ambiguïtés. Harris ne rejoint ce paradigme de l'entre-deux que dans la mesure où le génie de son anticipation prophétique est inséparable de la folie de sa "régression " païenne et de son anamnèse, celle qui fait suite à l'expérience de la transe-visitation et à l'engagement de l'homme fort usant de sa 
canne comme signe et instrument de puissance dans la lutte contre les fétiches, apportant la guérison miraculeuse par un baptême exorciste, attestant par sa polygamie des promesses de fécondité.

La fascination enchantée que peut exercer cet entre-deux hybride laisse place néanmoins, dans les relectures qu'en fait Marc Augé, surtout avec le recul historique et le passage de génération, à un diagnostic d'impasse : l'entre-deux prophétique se révèle en définitive un cul-de-sac. Le mimétisme par rapport au monde des Blancs n'a plus les vertus de la ruse symbolique et des rituels d'inversion/perversion. La répétition du rituel l'emporte sur les promesses du recommencement. Les voies hésitantes de l'imagination prophétique s'enferment dans une relation duelle Blanc/ Noir qui n'arrive pas à se dialectiser, le mouvement circulaire de retour à l'entre soi de l'origine efface l'acte inaugural du franchissement des frontières. Les synthèses imaginaires du Diable et du bon Dieu, de la confession et de l'accusation, ne remplissent plus en un mot la fonction symbolique des dispositifs rituels.

C'est au cœur même du "dispositif rituel restreint » qu'émerge l'analyse la plus systématique des impasses de cet entre-deux prophétique. L'institution thérapeutique de Bregbo et son rituel de la " confession diabolique » illustrent - à vrai dire dès l'époque du terrain alladian (et grâce aux ressources de l'ethnologie) - les promesses non tenues de cet imaginaire prophétique. Les « confessions diaboliques » sont pourtant l'illustration par excellence d'un bricolage inventif qui a fasciné toute une génération d'anthropologues. On peut même parler (pour reprendre le langage structuraliste de l'idéo-logique) de bricolage "syntagmatique " à propos de la " confession-ordalie " (Augé 1975 : 266-267), synthèse précipitée des chaînes de syntagmes des registres de la confession et de l'ordalie, de l'aveu de culpabilité et de l'accusation de l'autre persécuteur. Mais l'analyse anthropologique s'applique à démonter l'ambiguïté et d'une certaine façon à la lever jusqu’à produire le désenchantement et la perplexité, et même de sérieuses réserves.

La confession ordalique des sorciers présumés relève de l'aveu provoqué et arraché par la médiation du prophète répondant à l'accusation des autres, la famille de la victime, et elle n'est jamais séparée de l'accusation en retour des complices de l'" agir en diable». Les "confessions diaboliques » qui semblent faire plus de place à la spontanéité de l'aveu, pourraient représenter une vraie "conversion", mais ces confessions sont le fait de sorciers-victimes ayant trouvé plus forts qu'eux et conduits à avouer leur échec pour résoudre leur problème. Le dispositif rituel de ces confessions publiques et spectaculaires comporte donc des retournements significatifs de la logique sorcellaire sur elle-même, prise à son propre 
piège, mais on ne voit jamais pointer le message chrétien selon lequel les sorciers sont d'abord ceux qui croient à la sorcellerie, à commencer par les contre-sorciers. La conclusion est sans appel: la conscience du mal n'est pas transformée, la prégnance du schème persécutif reste entière, $y$ compris au cœur de la confession, au point de prendre la forme d'une "auto-accusation" qui peut se révéler morbide et mortifere si elle n'embraye pas sur les voies de la repentance et du pardon. On peut s'interroger, comme le fait Marc Augé, sur le présupposé évolutionniste, plus oedipien que wéberien, qui fait de la culpabilisation la voie obligée de l'individualisation. L'analyse "clinique» d'András Zempléni porte une attention plus généreuse aux voies du dédoublement du moi et aux opportunités d'une transition du registre de la persécution à celui de la culpabilité dans le procès d'individualisation. Dans ce «tribunal de la conscience " qu'instaure la culpabilité, le moi reste divisé intérieurement et soumis à des désirs contradictoires, mais la solution "chrétienne» consistant à inviter les sujets à renoncer à l'exutoire de l'accusation, à trouver en eux-mêmes l'énergie nécessaire pour assumer à titre personnel leurs pulsions agressives, en un mot à se prendre en charge, a du mal à se faire entendre. Le scénario du « je est un autre », l'oscillation entre l'autre maléfique qui me persécute (mon diable) et la part mauvaise de soi qui me culpabilise, n'aboutit pas à une autre conclusion. Le paradoxe d'un dispositif de confession qui fonctionne à l'accusation réussit à brouiller les pistes en faisant "l'économie de la culpabilité». La grille diabolique forme même comme "un rempart contre l'intériorisation de la culpabilité " (Zempléni 1975 : 209) et le procès de l'individualisation se fait attendre.

\section{Entre pardon et délivrance : guérir la nation}

Trente ans plus tard, ce détour anthropologique reste d'une étonnante actualité, y compris au regard des dispositifs de délivrance qui font le succès des pentecôtismes particulièrement au Brésil ou en Afrique. À sa façon, le pentecôtisme court-circuite le contrat éthique de la modernité religieuse, mais il importe plus que jamais de distinguer la logique de l'imaginaire païen qui nourrit le discours prophétique et les ressorts anthropologiques des dispositifs rituels qui convoquent le diable.

Le changement de contexte est manifeste. Les « nouveaux prophètes » de la ville et du monde qui se bousculent sur la scène ivoirienne, comme ailleurs, à partir des années 1990, sont moins que jamais marginaux, ils constituent d'authentiques révélateurs de l'évolution actuelle de l'Afrique. La religion de conversion est, pour reprendre les termes wéberiens, une "religion du tout de la vie", qui suppose l'implication dans toutes les 
dimensions de la vie personnelle et collective. Plus question de ces cohabitations tranquilles dont s'accommode le catholicisme dans ses rapports avec les pratiques païennes. La vocation des pasteurs prophètes s'inscrit dans une histoire personnelle d'expérience de "ressuscité», de guérison miraculeuse, qui se donne explicitement comme le paradigme des voies du salut et de la délivrance collective. Si le prophète, selon la formule de Marc Augé est «celui qui fait de sa vie un signe pour tous » (1982: 295), tous les récits de ces prophètes pasteurs, à l'image de leurs inspirateurs anglophones (Graham, Hagin, Osborn, Yonggi Cho, Idahosa) en témoignent.

Ces prophètes «de toutes les nations" disposent à la fois d'un fort ancrage national et d'un réseau international. L'idée de nation biblique, de peuple de Dieu, est au cœur de leur entreprise missionnaire transnationale et au fondement de la vision qu'ils ont du salut de leur pays. À défaut de fonder quelque Jérusalem céleste, le lieu imaginaire vers lequel leur regard est tourné et le miroir de la situation actuelle, c'est la Terre d'Israël. Les nouveaux hommes de Dieu n'investissent pas dans des lieux consacrés, le « site prophétique » est en premier lieu sur le net. Les assemblées qu'ils animent lors des cultes hebdomadaires s'accommodent de salons ordinaires, de salles d'arrière-cour, de garages ou d'entrepôts abandonnés, mais les grands rassemblements qu'ils organisent privilégient les lieux collectifs de rencontre (marché, stade, cinéma, salle municipale, ou chapiteaux). Dès que l'argent rentre, la construction d'un grand temple à étages mobilise tous les fidèles, mais faisant de nécessité vertu, cette religion de "parvenus » traduit par le choix de non-lieux religieux, sans histoire, sans identité, sans sacralité, à la fois un souci de déterritorialisation des pratiques religieuses et une politique d'occupation de l'espace public comme lieu d'enjeux du combat spirituel.

Une telle stratégie d'investissement de la scène médiatique et de "mise en spectacle » des séances de délivrance fait penser au "dispositif élargi », au sens où l'entend Marc Augé (1994: 94). Ces liturgies de rassemblement dans les stades, ces campagnes ou croisades d'évangélisation, ces démonstrations de guérisons miraculeuses, s'adressent moins à des communautés (ethniques ou territoriales) qu'à des assemblées d'individus (de frères et sœurs en Christ). Les prédicateurs étrangers invités attestent de l'importance des réseaux internationaux dans lesquels s'inscrivent les nouveaux ministères. Leur performance fait appel à l'art consommé de s'adresser à tous et à chacun, de faire face à une large audience collective, et de cibler individuellement le message ("Tu dois savoir que Dieu t'aime »; "Il y a ici quelqu'un qui...») en encourageant les témoignages. L'événement du rassemblement est démultiplié par le canal de l'appareillage audiovisuel qui 
fait partie du dispositif. Les grands pasteurs prophètes nigérians et ghanéens ont été les premiers à investir dans les entreprises de production de cassettes audio et vidéo, sans parler des chaînes de radio et de télévision.

Les forums nationaux de réconciliation ou de cérémonies du pardon, dont les prophètes pasteurs sont des acteurs, ont profondément modifié la donne des rapports du politique et du religieux. Si dans le contexte africain l'émergence de partis évangéliques et leur participation en tant que tels au jeu électoral n'est pas à l'ordre du jour (à la différence de l'Amérique latine), on sait par contre à quel point le discours évangélique et sa rhétorique informent le discours politique, et l'enjeu que représente le relais des campagnes d'évangélisation et la mobilisation des pasteurs dans l'élection d'un président "chrétien " (born-again). L'époque précédant les années 1990 où le pouvoir ivoirien pouvait se permettre de cantonner la parole et l'action des prophètes dans leur enclos thérapeutique, en pratiquant à bonne distance des liens de complicité et de soutien réciproque, est bien révolue. Le président reçoit officiellement les pasteurs nationaux et internationaux qui ne manquent pas de lui rappeler qu'un homme politique doit avoir "une vision». La présence attitrée d'un pasteur conseiller, expert et négociateur, et éventuellement marchand d'armes, auprès de la présidence, prend le relais des marabouts du prince ${ }^{8}$. Le couple présidentiel peut accompagner de sa présence l'accueil, dans le stade Houphouët-Boigny, d'un pasteur coréen comme Yonggi Cho prophétisant la réussite d'une Côte-d'Ivoire délivrée de ses démons. Le message de délivrance et de prospérité formulé par Isaïe (Livres prophétiques, 45), que le président déclare avoir "reçu » dès son investiture, et qu'il lit et relit régulièrement, est censé servir de guide et de promesse au nouveau Cyrus, chargé de terrasser les nations rebelles, de libérer les captifs, et de reconstruire la maison de Dieu. Mais la conversion prophétique du politique qui passe par la médiation du couple de l'homme d'État, sauveur providentiel, et du prophète de Dieu comporte tous les pièges d'une relation duelle et spéculaire qui finit par engendrer une synthèse imaginaire de la pensée appliquée et de l'action inspirée.

8. Le gourou de la famille Gbagbo, bien connu aujourd'hui des médias, était aux dernières nouvelles le "Révérend » Koré Moïse, apôtre et prophète d'une assemblée de la Mission internationale Schekina Glory d'origine américaine. Cet ancien «ingénieur » en télécommunications, international de basket et président de la fédération ivoirienne, récuse sa réputation de "pasteur de Ggagbo » : "Je suis le pasteur d'une Église de trois mille membres, et Laurent Gbagbo en fait partie. Je ne me suis jamais présenté comme le pasteur particulier de Gbagbo" (Le Nouvel Afrique-Asie, "Top Info ", 7 octobre 2004). 
Le prophétisme pentecôtiste véhicule les schèmes symboliques disponibles de l'imaginaire du mal dans les diverses sphères de l'existence: de la "renaissance » à la « délivrance». Les dispositifs rituels qu’il inspire (confessions, témoignages, exorcismes, guérison miraculeuse) informent le discours politique et créent des effets discursifs qui dérèglent la syntaxe du dicible, déplacent les limites du possible et du pensable. Dans son premier discours à la Nation ivoirienne, aux lendemains de l'entrée en guerre des « rebelles du Nord» en septembre 2002, le président Laurent Gbagbo terminait sur ces paroles: "Que le Dieu Tout-Puissant qui nous a créés et qui a mis chacun à sa place; Que le Dieu Tout-Puissant qui fait la paix, qui fait aussi la guerre, que le Dieu Tout-Puissant qui sait tout ce qui s'est passé et tout ce qui arrive, qu'il bénisse la Côte-d'Ivoire et qu'il nous libère des méchants». Le même homme inaugurait en octobre 2001, le forum de réconciliation "Vérité, repentance et pardon» censé, entre autres, lever le mal du charnier de Yopougon, par d'autres paroles plus «évangéliques »: "Dites seulement une parole, et la Côte-d'Ivoire sera guérie». Le recours aux rhétoriques du pardon et de la réconciliation et les institutions juridiques d'amnistierepentance, qui font l'économie de la vérité et de la justice au profit de la paix, vont parfaitement de pair avec les idiomes politiques de la guerre spirituelle et l'offre religieuse de dispositifs de délivrance.

Les promesses des dispositifs élargis de la délivrance et du pardon doivent être jugées au regard du traitement des altérités dont ils sont porteurs. Dans le christianisme, la grâce du pardon s'est imposée comme la voie royale de l'apaisement des cœurs et de la délivrance du mal. En soulignant que « le pardon est une destruction de la faute sans la destruction du coupable», Robert Hertz (1988 [1922] : 56) resitue néanmoins «le mystère du pardon » dans une généalogie de mécanismes spirituels où l'effacement du mal passe soit par la vengeance plutôt que par la clémence (ou la faiblesse du pardon), soit par l'« exorcisme ", l'expulsion ou la destruction de l'agent maléfique. Dans ce dernier sens, l'esprit de la délivrance est clairement le contraire de l'esprit du pardon puisqu'il invite à s'engager, sans compromis ou renoncement, dans la guerre contre le mal, à répondre au mal par le mal. Prier pour le pardon de Dieu a pour envers, du moins pour les pasteurs ou prophètes de la mouvance évangélique et pentecôtiste, la lutte déclarée contre les démons. La guerre spirituelle passe par l'élimination de l'autre méchant qui vous veut du mal. Dans les "guerres de pasteurs " dont se nourrissent régulièrement les médias (au Nigeria comme en Côte-d'Ivoire), l'accusation de recours aux pratiques fétichistes (vodou «béninois » ou autres) à des fins de pouvoir participe du jeu de l'accusateur accusé et confirme l'emprise de l'autre païen au cœur des Églises. 
Dans une mouvance, qui conjugue de longue date (particulièrement dans la culture américaine) l'évangélisme avec le patriotisme, l'accusation à peine euphémisée des ennemis antipatriotes de l'intérieur et de l'extérieur assimile les mauvais citoyens et les migrants qui se mêlent aux gens du Nord. Si les démons restent le fétichisme et la sorcellerie des autres avec lesquels "pactisent " les Églises païennes, le danger suprême aujourd'hui pour la mouvance évangélique, en Côte-d'Ivoire comme ailleurs, est bel et bien l'islam "diabolisé " par les pasteurs. Un dispositif élargi comme le «forum des religions » opère sur un fond d'œcuménisme et de tolérance, tout en pratiquant une discrimination subtile vis-à-vis de ceux qui seraient tentés de vouloir relancer ou exploiter la guerre des religions et l'opposition entre un Nord musulman et un Sud chrétien ${ }^{9}$. Le paradoxe de ce "procès » est qu'il repose sur la diabolisation non d'un islam extrémiste ou fondamentaliste, mais au contraire d'un islam réformiste plutôt respectueux du pluralisme local des traditions musulmanes et ouvert sur la modernité, un islam qui, pour des raisons d'intérêt évidentes, est la seule composante religieuse à soutenir pleinement le principe de la laïcité de l'État ivoirien ${ }^{10}$.

La promotion des dispositifs narratifs de la délivrance au rang d'idiome politique conduit à s'interroger sur le projet communautaire d'une mouvance religieuse qui pour sauver la Côte-d'Ivoire de ses maux, et la gagner à Jésus, compte exclusivement sur le Peuple de Dieu, « le vrai Peuple de la Nation", et fait peser le soupçon, à défaut de l'accusation, sur les étrangers, les inconvertis (les païens, les catholiques, et les musulmans). Par son aspiration à un État délivré enfin du mal de la division, sa confiance dans une puissance de l'Esprit opérant sans médiation, son souci de porter témoignage de l'unité organique du Peuple de Dieu, le discours théocratique du pentecôtisme fait peu de cas du traitement des altérités et se révèle complice des impasses identitaires de l'ethno-nationalisme.

\section{La délivrance : l'envers du spectacle}

L'imaginaire de la délivrance des forces du mal peut redonner de la force au discours politique mais pour juger de sa "performance», il importe de revenir sur les dispositifs cultuels restreints qui conferent toute leur

9. Nous nous référons particulièrement ici à un débat diffusé le 17 juillet 2001 sur TVI.

10. Marie Miran (2000 : 145) observe que la formation du Conseil national islamique en 1993 à l'initiative d'étudiants, intellectuels ou cadres «réformistes" refusant l'assignation à un islam ethnique, en l'occurrence dioula, est aussi une réponse à l'agressivité croissante des pratiques d'évangélisation des Églises protestantes. 
crédibilité aux hommes forts que sont les prophètes pasteurs. La rencontre entre les hommes politiques et tel ou tel prophète pasteur passe souvent par une histoire familiale de conversion et de guérison où se nouent la confiance personnelle et le crédit prophétique. En prenant le relais des villages thérapeutiques, les centres de délivrance (Fancello 2006) créés par les prophètes pasteurs accompagnent un procès de conversion qui continue à lier la guérison aux satisfactions régressives de l'accusation de l'autre dévorant et mortifere. Le regain de force promis aux individus se nourrit des ressorts de la diabolisation de l'autre proche qui vous "attache" et plus globalement de la "satanisation " des esprits vampireux de la famille. La planétarisation du combat contre les forces du mal se replie sur les rancœurs du microcosme familial.

Les séances collectives et régulières de délivrance font peu de place, il faut le dire, à un traitement individualisé, à l'écoute des problèmes. La maîtrise des sujets agités par des équipes d'auxiliaires usant de techniques du corps appropriées, le regroupement collectif, devant l'autel, de la masse des corps "tombés en esprit » et affalés sur le sol, ou le corps à corps du pasteur avec les possédés relèvent plus de la performance théâtrale que de la "cure d'âmes». À l'exemple de l'évolution du pentecôtisme brésilien, certains pasteurs ne descendent plus dans l'arène, et se livrent au traitement didactique de cas présélectionnés en procédant à un questionnement édifiant qui offre au public une sorte de leçon d'introduction, par l'exemple, au monde des esprits. Quant les esprits sont trop nombreux pour être appelés à se manifester et être "attachés » en une seule soirée, on se réserve pour une autre séance, avec la complicité du "possédé». Dans cette délivrance didactique, c'est seulement une fois revenu à lui-même que le sujet raconte les circonstances et le contexte de sa "possession", en évoquant les complices de son envoûtement. Le "possédé» qui vient d'étaler en public (et parfois devant la caméra) les drames familiaux les plus intimes, retourne simplement à sa place, dans l'anonymat le plus complet et l'indifférence générale des frères et sœurs en Christ qui réservent leur énergie pour la louange. Cette théâtralisation didactique de la délivrance à la brésilienne évoque ce que Thomas Csordas appelle la "domestication de la délivrance », une « distanciation esthétique " par rapport à la "performance rituelle » qui permet le contrôle de l'engagement émotionnel et répond à l'attente "psycho-culturelle» des classes moyennes américaines (Csordas 1994 : 179).

L’originalité des dispositifs de délivrance, brésiliens ou africains, repose sur le bricolage inventif des registres de la possession démoniaque, de l'accusation de sorcellerie et de la confession en double ou en diable. Les média- 
tions théâtrales et symboliques de la possession provoquée par l'imposition des mains ne se contentent pas de convoquer quelque panthéon local de dieux ou de génies; elles n'excluent pas en effet la désignation publique et nominale, plus ou moins directe, des personnes de l'entourage de la victime (sœur, oncle, époux) et la désignation d'esprits plus directement familiaux : l'esprit de la mère jalouse qui veut dévorer son enfant ; le père incestueux qui joue les «maris invisibles» avec sa fille; les parents qui sacrifient leur fils au vodou; l'enfant-sorcier qui bloque la réussite professionnelle du père de famille. La nomination de l'autre sorcier est le gage de sa "manifestation » et la condition de son "détachement». Les témoignages publics, et enregistrés, qui feront suite à la séance de délivrance (certains relayés par la radio) placent les affaires de famille ou de voisinage sous le regard des projecteurs et conferent au dispositif une dimension médiatique (qui n'est peut-être pas la meilleure voie de la médiation familiale).

La mise en scène de l'exorcisme joue sur le dédoublement de la personne possédée pour ménager en fait la cohabitation de l'accusation de l'autre qui vous possède (le diable c'est lui, l'autre proche) et de l'aveu du coupable qui parle par la voix du possédé (l'acteur en diable). Dans ce double (ou triple) jeu, ni la responsabilité de l'accusation, ni la culpabilité de la confession ne sont vraiment assumées («je»parle par un autre). Personne dans ce dispositif n'est directement accusé ou accusateur : le pasteur se garde bien de désigner qui que ce soit en personne puisqu'il se contente de faire pression pour que le mauvais esprit se "manifeste»; la victime n'est pas censée assumer les paroles émises par celui qui l'habite ; et la personne qui avoue sa jalousie, le double diabolique qui habite le corps et avec lequel le pasteur engage le dialogue, n'est pas vraiment coupable. Comme dans les " confessions en diable » organisées par le prophète Atcho de Bingerville, le dispositif permet, par le jeu du dédoublement, à la fois d'euphémiser l'accusation et de faire l'économie de la culpabilité. Pas question, même dans ce contexte chrétien, de confession accompagnée de contrition ou de repentir personnel, et encore moins de pardon demandé ou accordé à l'autre. La solution c'est l'exclusion de l'autre, imaginaire et mortifere, des corps et des lieux.

\section{Conclusion : le triangle anthropologique de la persécution}

Les récits de guérison des sujets possédés ou ensorcelés qu'on peut entendre dans les Églises pentecôtistes sont loin d'évoquer, à la manière des initiés des cultes de possession, quelque joyeuse cohabitation des contraires: ils témoignent d'un moi divisé, travaillé par le dualisme du bien et du mal, ou tout du moins par des forces opposées qui font obstacle 
à l'idéal chrétien d'un moi unifié et transparent. L'ambivalence malheureuse de leurs sentiments est faite d'un mélange d'agressivité et de culpabilité vis-à-vis d'un entourage familial auquel on est censé tout devoir et donner toute sa confiance et dont on craint la jalousie et la duplicité perverse. La sorcellerie est l'envers d'un ethos de la dette communautaire qui pèse d'autant plus fortement sur les sujets d'aujourd'hui que les conditions matérielles et sociales de la vie actuelle dans les capitales africaines permettent de moins en moins aux jeunes salariés, menacés par le chômage, de répondre aux attentes de redistribution des vieux du village ou des parents proches.

À défaut d'éveiller la conscience sociale et politique des masses, le manichéisme du combat entre Christ et Satan est censé offrir des bénéfices psychologiques à des individus en proie à l'ambivalence incontrôlable de leurs sentiments. Le converti est invité, à l'image du prophète-pasteur, à être un entrepreneur, un vainqueur, et pour cela à faire preuve d'une agressivité assumée contre le mal. Si on peut admettre que cette agressivité retrouvée soit une source d'énergie (empowerment), et que le prophète joue un rôle d' « embrayeur" de violence, il faut aussi reconnaître que celle-ci a un coût pour les familles autant que pour les sujets. La vampirisation des liens de sang à laquelle se livrent les apprentis-sorciers que sont les pasteurs transforment la vie de famille des convertis en un enfer qui n'est pas censé avoir d'autres solutions que la conversion de tous dans le "cercle du sang de Jésus». La multiplication de la précarité des situations dans le monde urbain ne permet pas à l'inverse de céder à l'individualisme et de s'offrir le luxe d'une rupture avec la famille qui reste le seul capital et la seule ressource en dernière instance dans une économie de survie. Le compromis entre les aspirations individuelles à l'autonomie et la gestion responsable de la dette familiale s'impose (Marie 1997 : 303). La rupture consommée avec les "vampires familiaux " peut miser sans doute sur les ressources de la nouvelle famille des frères et sœurs en Christ, mais les risques d'assujettissement à une nouvelle communauté pèsent aussi sur les nouveaux "sujets de Dieu » dont la dette infinie vis-à-vis du Père prend le relais de la dette de sang des parents.

La précipitation à inscrire le pentecôtisme dans les grands récits de la modernité ferait volontiers passer l'Esprit Saint pour un agent du sujet moderne, oubliant un peu vite que l'agent double de la conversion c'est le diable. La diabolisation du sorcier consacre une exacerbation et une dérégulation de la logique païenne plus qu'elle ne témoigne de sa plasticité. Dans ce triangle anthropologique du paganisme, du prophétisme et du 
pentecôtisme, nul doute que le schème de la persécution occupe une place centrale qui engage les catégories de la personne. On ne peut néanmoins que s'interroger sur le basculement qui conduit le même schème de l'accusation de l'autre à fonctionner, dans le contexte enchanté du paganisme, au service de la pluralité de la personne, de l'expression de son ambivalence, et de sa réintégration sociale dans un esprit de tolérance, et à l'inverse, pris dans l'engrenage du prosélytisme missionnaire, soumis au manichéisme du bien et du mal, à se mettre au service d'une logique régressive d'exclusion et de dépendance.

La globalisation de la sorcellerie et la promotion de la grille diabolique à l'échelle mondiale n'ont pas automatiquement pour conséquence la libération des individus des enclos communautaires et leur transmutation comme simples sujets de Dieu. La reconversion de la culpabilité individuelle, suscitée par le désir d'indépendance, en agressivité retournée contre tel ou tel membre de la famille et transmuée en persécution, est-elle bien la voie d'un travail sur soi et d'une conversion du sujet à lui-même? En donnant toute sa caution au schéma persécutif, en confortant l'idée que l'enfer c'est les autres, les méchants, on ne sort pas vraiment les individus de leur dépendance et de leur aliénation. Les promesses d'ouverture sur une nouvelle subjectivité, morale ou politique, que comporterait l'émergence des pentecôtismes relèvent du discours prophétique.

Centre national de la recherche scientifique, Paris Centre d'études interdisciplinaires des faits religieux amary@ehess.fr

MOTS CLÉS/KEYWORDS : paganisme/paganism - pentecôtisme/pentecostalism - sorcellerie/witchcraft - christianisme africain/african christianism - prophétisme/prophetism. 
Augé, Marc

1975 Théorie des pouvoirs et idéologie. Paris, Hermann.

1979 Symbole, fonction, histoire. Paris, Hachette.

1982 Génie du paganisme. Paris, Gallimard.

1986 Un ethnologue dans le métro. Paris, Hachette.

1994 Pour une anthropologie des mondes contemporains. Paris, Aubier.

1997 La Guerre des rêves. Exercices d'ethnofiction. Paris, Le Seuil.

Augé, Marc \& Jean-Paul Colleyn

1990 Nkpiti : la rancune et le prophète.

Paris, Éd. de l'Ehess.

Balandier, Georges

1955 Sociologie actuelle de l'Afrique Noire. Paris, Presses universitaires de France.

Bessis, Raphaël

2004 Dialogue avec Marc Augé. Autour d'une anthropologie de la mondialisation. Paris, L'Harmattan.

\section{Csordas, Thomas}

1994 The Sacred Self. A Cultural

Phenomenology of Charismatic Healing.

Berkeley, University of California Press.

Dozon, Jean-Pierre

1995 La Cause des prophètes. Politique et religion en Afrique contemporaine. Postface de Marc Augé. Paris, Le Seuil.

Fabian, Johannes

1971 Jamaa: A Charismatic Movement in Katanga. Evanston, Northern University Press.

\section{Fancello, Sandra}

2006 Les Aventuriers du pentecôtisme ghanéen. Nation, conversion et délivrance en Afrique de l'Ouest. Paris, IRD-Karthala.

\section{Hertz, Robert}

1988 [1922] Le Péché et l'expiation dans les sociétés primitives. Paris, Jean-Michel Place.

Lévi-Strauss, Claude

1962 La Pensée sauvage. Paris, Plon.

Marie, Alain

1997 «Avatars de la dette communautaire. Crise des solidarités, sorcellerie et procès d'individualisation (itinéraires abidjanais) ", in Alain Marie, ed., L'Afrique des individus. Paris, Karthala : 249-328.

\section{Mary, André}

1993 «Le travail symbolique des prophètes d'Eboga : logiques syncrétiques et entredeux culturel ", Cahier d'Études Africaines, 132 (33) : 613-643.

2002 «Prophètes-pasteurs. La politique de la délivrance en Côte-d'Ivoire ", Politique Africaine 87 : 69-94.

Miran, Marie

2000 «Vers un nouveau prosélytisme islamique en Côte-d'Ivoire : une révolution discrète ", Autrepart 16 : 139-160.

Peel, John

2000 Religious Encounter and the Making of the Yoruba. Bloomington-Indianapolis, Indiana University Press.

Piault, Colette, ed.

1975 Prophétisme et Thérapeutique. Albert Atcho et la communauté de Bregbo. Paris, Hermann.

\section{Shank, A.}

1994 Prophet Harris. The "Black Elijah" of West Africa. Leiden, Brill.

\section{Zempléni, András}

1975 "De la persécution à la culpabilité », in Colette Piault, ed., Prophétisme et Thérapeutique... : 153-219. 